\section{Reviewers' reports should in turn be peer reviewed}

SIR - As a young investigator with limited experience in the peer-review process, I would like to add a perhaps naive suggestion to the comments made by Rory Wilson (Nature 441,812; 2006) and others in Correspondence and at www.nature.com/ nature/peerreview/debate/index.html).

As a result of my conversations with peers and mentors, I suggest that, when assessments are complete, journals ask reviewers to review the other reviewers' comments before the editor makes a decision about publication. Although this assessment is traditionally reserved for the editor, by holding reviewers accountable, thereby encouraging fair and reasonable reports, editors will be better able to assess the suitability of a recommended rejection, revision or acceptance. I believe my suggestion would be particularly useful in journals for which the editor is a practising scientist. In these cases the editor is not anonymous, so may not assess reports as freely as reviewers who have this protection.

Although the time taken for the initial review process may be increased by my suggestion, I believe it would result in a fairer process, as the editor would benefit from the feedback in the decision-making stage and the reviewers would be given an incentive to provide their services fairly.

Alexandra List

Department of Psychology, University of Califomia, Berkeley, California 94720-1650, USA See Nature's web debate on peer review at www. nature.com/hature/peerreview/debate/index.html

\section{Judge a paper on its own merits, not its journal's}

SIR - Your News story ${ }^{\alpha}$ Cash for papers: putting a premium on publication" (Nature 441,$792 ; 2006$ ) reports that South Korea is joining China and Pakistan in rewarding researchers with cash for publications in élite journals. Presumably the higher the journal's impact factor, the more valuable the reward.

The impact factor may be a good indicator of the quality of a journal, but it is misleading to judge an individual publication by the name of the journal in which it appeared. A journal's impact factor, as defined by the ISI, is the average number of citations per article the journal received during a given period. Isn't the number of citations a paper receives a better, more direct measure than the average of papers in the same journal?

A journal is good because it contains many high-impact papers, but not all papers in that journal necessarily have high impact. Papers that are published in low-profile journals but receive an equally high number of citations should not only be rewarded, but be rewarded more. In judging an individual publication, what counts should be its real merits, not its batch label.

Shu-Dong Zhang

MRC Toxicology Unit, Hodgkin Building, Lancaster Road, Leicester LE1 9HN, UK

\section{Unpredictable Sun leaves researchers in the dark}

SIR - We agree with the view expressed in your News Feature ${ }^{\alpha}$ The dark side of the Sun" (Nature 441, 402-404; 2006) that solar magnetic activity is important. It leads to such dangerous events as solar flares and coronal mass ejections, as well as being associated with fascinating phenomena such as sunspots and the solar cycle.

The Sun's magnetic field is now believed to be generated by a hydromagnetic dynamo acting deep within its interior, where streams of highly ionized plasma generate electric currents and, in turn, magnetic fields. Progress in dynamo theory is extremely difficult, as it can be made only by understanding the interaction of turbulent plasma motions with magnetic fields. Indeed, the extreme conditions within the solar interior make this a formidable task; understanding even the much less turbulent environment of the Earth's atmosphere stretches current theories to their limits, and weather prediction is notoriously precarious.

It is in this context that current attempts to predict magnetic cycles on the Sun must be viewed. The model proposed by Mausumi Dikpati and her team, highlighted in your News Feature, relies on parametrization of many poorly understood effects. Although such parametrized models have been widely (and legitimately) used to explore specific features of dynamo processes, they have no detailed predictive power. Indeed, there is vociferous debate in the field, not just about the size of many of the effects included in Dikpati's (and many other people's) models but even their signs. Moreover, the dynamo equations are extremely nonlinear; the solar dynamo is believed to exist in a state of deterministic chaos, making prediction intrinsically yet more difficult. Any predictions made with such models should be treated with extreme caution (or perhaps disregarded), as they lack solid physical underpinnings.

Of course it is interesting to speculate on what direction solar magnetic activity might take in the future. Recent sunspot cycles have been exceptionally vigorous, as noted by S. K. Solanki and colleagues (Nature 431, $1084-1087 ; 2004)$. It is well known that, in the past, such episodes of high activity have tended to be followed by a dramatic crash into periods of severely reduced magnetic activity, termed Grand Minima. Although we would not presume to predict that this will happen soon, it would certainly be interesting to witness such a collapse.

Steven Tobias ${ }^{\star}$, David Hughes ${ }^{\star}$, Nigel Weiss $†$

*Department of Applied Mathematics, University of Leeds, Leeds LS2 9JT, UK

tDepartment of Applied Mathematics and Theoretical Physics, University of Cambridge, Cambridge CB3 OWA,UK

\section{Second thoughts on who goes where in author lists}

SIR - I thought I understood the guidelines for determining scientific authorship: the individual making the greatest intellectual contribution is the lead author, followed sequentially by those making progressively lesser contributions. In addition, the finalauthor slot is sometimes reserved for a lab head or project initiator, who may have made little direct contribution to the paper but deserves some vague honour nonetheless.

But now I am confused. A collaborator of mine at the University of Cambridge asked to be moved from second to last position on a four-authored paper. When I asked why, he said the British Research Assessment Exercise (RAE), which determines departmental rankings and government funding, gives greater credit to the final than even the second author on a multi-author paper. My confusion deepened when two other colleagues - both Americans — had a lively disagreement about who would be last author on a paper with seven authors.

The 'communicating' (or 'corresponding') author is often, but not always, the lead author. If he or she is not the lead, is some special significance attached to this? Does it count for something on the RAE? Some disciplines have evolved their own idiosyncratic rules. I have also noted that another common convention is to list authors alphabetically, but does the RAE know if Williams made a lesser or greater contribution than Anderson?

Is there a set of coherent authorship rules written down somewhere that, in my 20-year research career, I have managed to miss? If not, then perhaps there should be.

Please note that I am the first, last and communicating author on this Correspondence.

William F. Laurance

Smithsonian Tropical Research Institute, Apartado 2072, Balboa, Panama

Nature's most recent Editorial stating its policy on this perennial topic, "Games people play with authors' names" (Nature $387,831 ; 1997)$, can be seen at www.nature. com/nature/journal/v387/n6636/full/ 387831a0_fs.html - Editor, Nature. 